\title{
Anterior mediastinum invasion by multiple myeloma: A case report
}

\author{
YING-HUI XU ${ }^{1}$, LU-GUO SUN ${ }^{2}$, CHAO SUN $^{1}$, OU BAI $^{1}$, TING-TING LIANG $^{1}$ and KE-WEI MA ${ }^{1}$ \\ ${ }^{1}$ Cancer Centre of First Hospital of Jilin University, Changchun, Jilin $130021 ;{ }^{2}$ National Engineering Laboratory \\ for Druggable Gene and Protein Screening, Northeast Normal University, Changchun, Jilin 130024, P.R. China
}

Received August 16, 2014; Accepted April 29, 2015

DOI: $10.3892 / 01.2017 .5756$

\begin{abstract}
Multiple myeloma (MM) is a clonal proliferation of malignant plasma cells in the bone marrow (BM) that secretes monoclonal paraproteins in the blood serum and urine. Bone marrow MM cells can invade and damage the functions of other tissues and organs, such as the lungs, spleen, liver, pancreas, kidneys and lymph nodes. However, the invasion of MM cells primarily located in the BM to the anterior mediastinum at the site of the thymus is an extremely rare event. The current study reports the case of a 53-year-old female who presented with MM with involvement of the anterior mediastinum. The diagnosis was based on clinical imaging analyses and the results from BM and laboratory examinations, local biopsy pathology and immunohistochemistry. The patient was administered two courses of chemotherapy (epirubicin, dexamethasone and thalidomide). As a result, the tumor reduced in size, but the laboratory examination indicated no significant change. Next, the patient was switched to one course of PAD chemotherapy (bortezomib, epirubicin and dexamethasone). The original tumor was significantly reduced in size following this chemotherapy, and all the indicators improved. The present study suggests that invasion of the thymus by MM may lead to immune disturbance arising from the abnormal thymus gland. In the clinic, extramedullary plasmacytoma in the thymus should be carefully distinguished from thymoma.
\end{abstract}

\section{Introduction}

Multiple myeloma (MM) accounts for $13 \%$ of all hematological malignancies, with an annual incidence of 6.5/100,000 people in the Western world (1). MM occurs in middle-aged individuals, mostly between 50 and 60 years old. The incidence rate increases with age, and MM is rarely found in individuals in

Correspondence to: Dr Ke-Wei Ma, Cancer Centre of First Hospital of Jilin University, 71 Xinmin Street, Changchun, Jilin 130021, P.R. China

E-mail: makw@jlu.edu.cn/sunchao-347@163.com

Key words: multiple myeloma, anterior mediastinum, bone marrow, invasion their 40s. MM is a neoplastic plasma cell disorder that is characterized by the clonal proliferation of malignant plasma cells in the bone marrow (BM) and the secretion of a monoclonal paraprotein in the blood serum and/or urine (2). The monoclonal proliferation of plasma origin cells can be observed in extraosseous extramedullary plasmacytoma or in the context of MM.

MM cells that are primarily located in the BM may invade other tissues and organs, such as the lungs, spleen, liver, pancreas, kidneys and lymph nodes, leading to dysfunction of the corresponding organs. MM patients can develop clinical symptoms such as hypercalcemia, renal insufficiency, anemia and bone disease (2). However, to the best of our knowledge, the invasion of myeloma cells to the anterior mediastinum at the site of the thymus is extremely rare. The current study reports the case of a 53-year-old female who presented with MM with involvement of the anterior mediastinum. The characteristics and diagnosis of this case, and the therapeutic effect following conventional anti-MM therapy were analyzed. This study was approved by the Ethics Committee of the First Hospital of Jilin University (Changchun, China). Informed consent was obtained from the patient.

\section{Case report}

A 53-year-old female was referred to the Cancer Centre of First Hospital of Jilin University (Changchun, China) on September 4, 2013 with a visible protruding mass on the right chest wall accompanied with local pain. The patient stated that the mass had first been observed 2 years previously and had continued to grow since then. However, except for the feeling of fatigue, the patient presented with no other complaints, such as a fever, a cough or bone pain throughout the body; therefore, daily life was not affected. A physical examination showed a single, round, hard and egg-sized neoplasm protruding from the upper right chest wall (Fig. 1). No superficial lymphadenopathy was present, with the exception of mild anemia.

The patient presented to Inner Mongolia People's Hospital (Hohhot, China) in August 2013. A tumor with dimensions of $48.7 \times 60 \times 52 \mathrm{~mm}$ was detected in the right anterior mediastinal region by computed tomography (CT) scan and was diagnosed as a malignant thymoma. After 1 week, the patient presented to the Chinese Academy of Medical Sciences Cancer Hospital (Beijing, China) for further diagnosis. On August 12, 2013, 

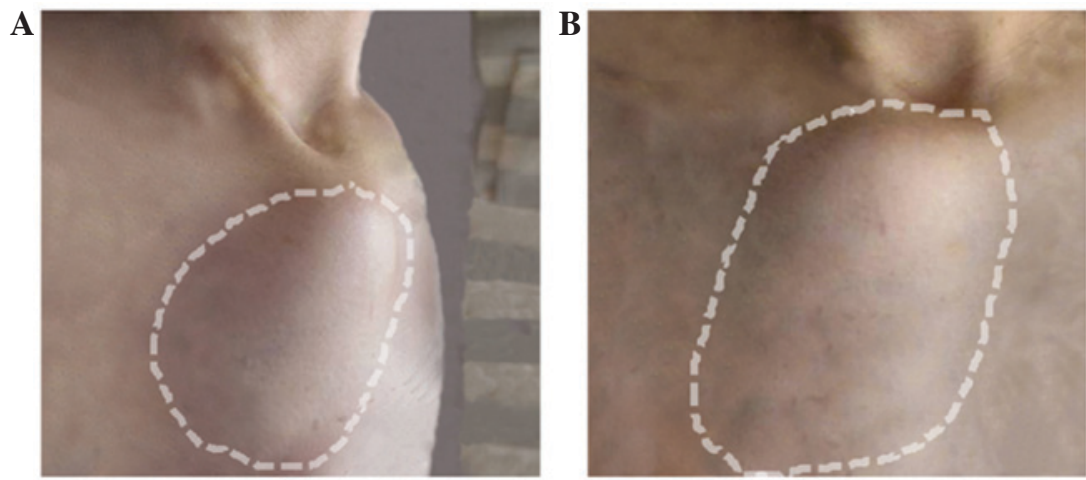

Figure 1. Macroscopic observation of the mass on the upper right chest wall of the female patient. (A) Lateral and (B) front view.
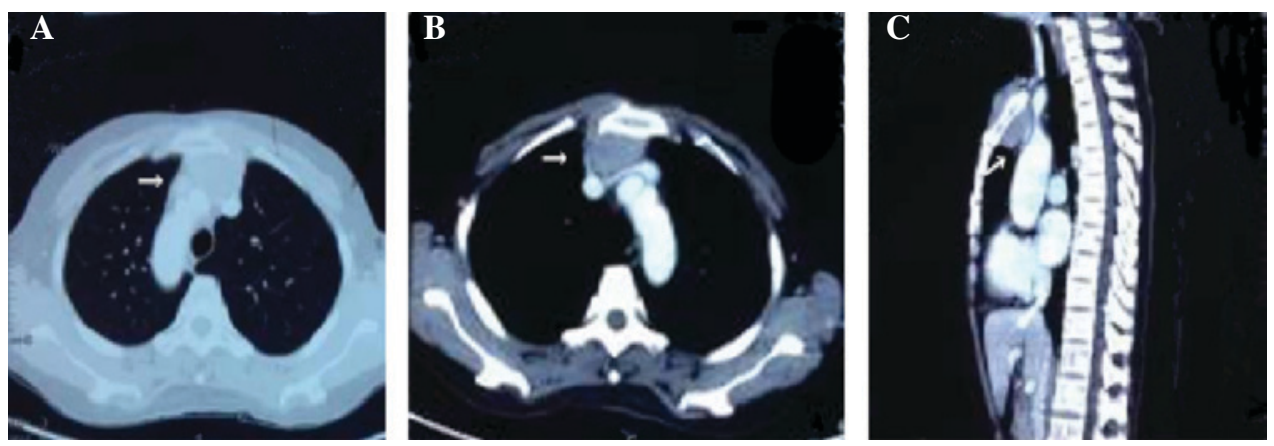

Figure 2. Imaging examination prior to therapy: Chest computed tomography scan prior to therapy. The arrows indicate the mass in the region of the thymus in the anterior mediastinum at the right chest wall. (A) Pulmonary window, (B) mediastinal window and (C) sagittal view.

a CT scan showed an aggressive tumor in the right anterior mediastinal region. Further biopsy and cytopathological analyses revealed expansion of cortical thymoma and a large number of lymphocytes distributed between epithelial cells, which suggested a thymoma with lymphocytes. The histopathological analysis in August 14, 2013 revealed several fibrous tissue squeezed and degenerated with infiltrations of inflammatory cells, indicating a proliferation of lymphocytes, and thus excluding a diagnosis of thymoma with lymphocytes.

In September 2013, the patient presented to the First Hospital of Jilin University for an imaging examination. A chest CT scan (Fig. 2) revealed a large, round mass, $48.7 \times 60 \times 52 \mathrm{~mm}$ in size, in the region of the thymus in the anterior mediastinum at the right chest wall. Head magnetic resonance imaging, abdominal $\mathrm{CT}$ and bone scans appeared normal. Based on the imaging data, the mass was suspected to be a malignant primary or metastatic tumor.

To clarify the origin of the mass, a pathological analysis of the neoplasm biopsy was performed, which found a plasmoma with invaded striated muscle (Fig. 3). Further immunohistochemistry (IHC) examinations revealed that the tumor was positive for Ki-67 (+30\%), cluster of differentiation (CD)38, CD138, immunoglobulin (Ig) light chain $\lambda$ (LAM) and multiple myeloma oncogene-1 (MUM-1) (Fig. 4), and negative for B-cell lymphoma (Bcl)-2, Ig light chain $\kappa(\mathrm{KAP}), \mathrm{CD} 20$, CD79a, CD3, CD43, cytokeratin (CK), CK19, terminal deoxynucleotidyl transferase, CD1a, myeloperoxidase, CD68, CD5, cyclin D1, CD10, Bcl-6, CD21 and CD23. A BM biopsy was then performed, which found that the proportion of plasma cells was extremely high and that the ratio of primitive to

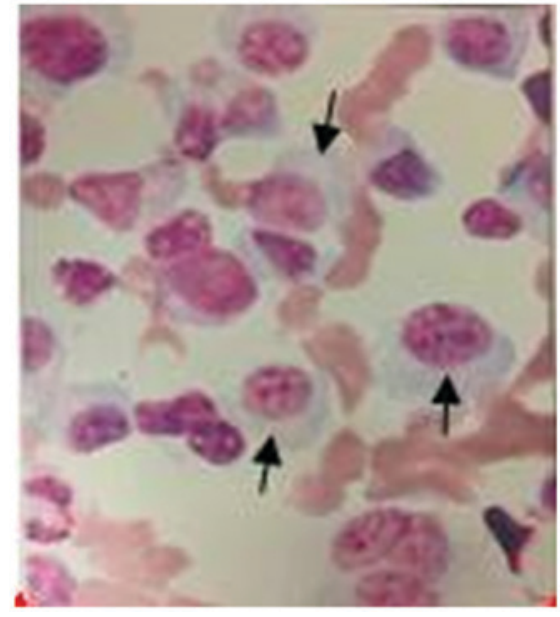

Figure 3. Pathological test results of the bone marrow, with arrows indicating malignant plasma cells.

naive plasma cells reached $57 \%$, with a tendency to group in a clustered arrangement. Additional flow cytometry analyses of BM aspiration showed that p15-positive cells accounted for $33.11 \%$ of the cells, with high positivity for CD38, CD138, CD9 and CD28, positivity for LAM, and native results for KAP, CD7, CD34, CD117, CD10, CD33, C-X-C chemokine receptor type 4, CD200, CD56, CD19, CD123, CD13, CD22, CD20 and CD45.

In conventional blood tests, the reticulocyte (red blood cell) count $\left(3.33 \times 10^{12}\right.$ cells/1; normal range, 3.80$5.10 \times 10^{12}$ cells/l) and hemoglobin (95 g/l; normal range, 

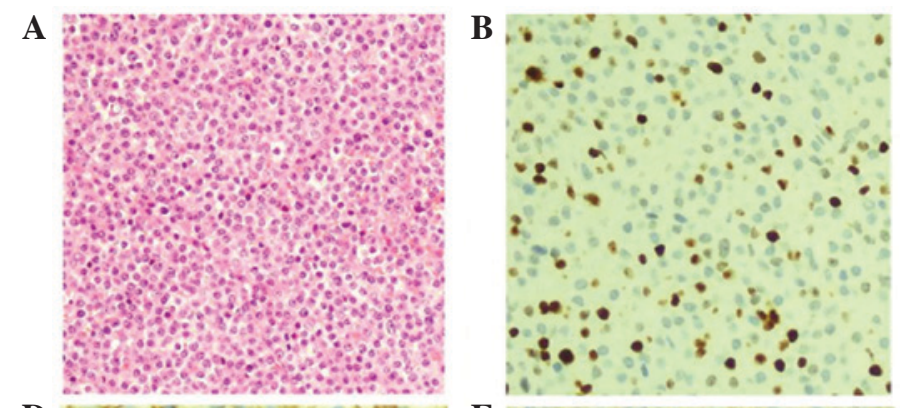

D

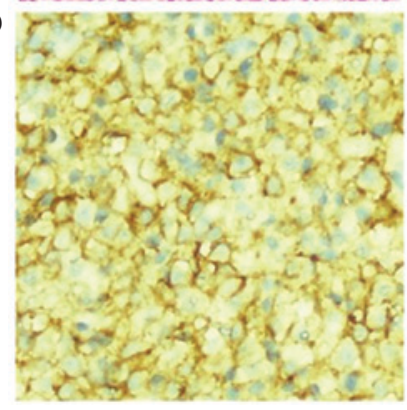

E

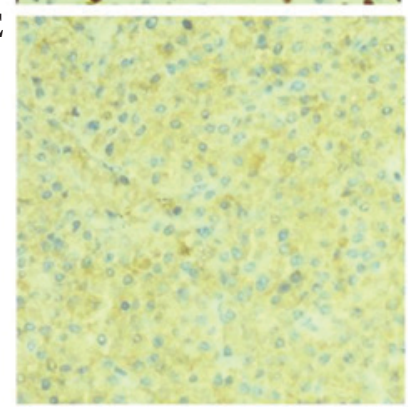

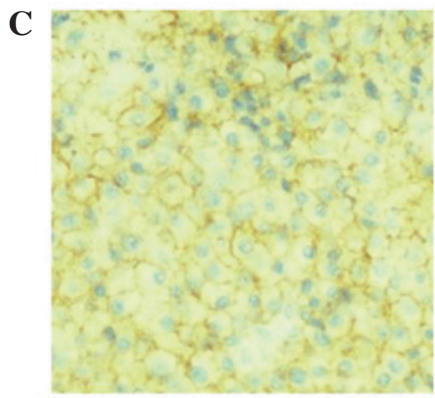

F

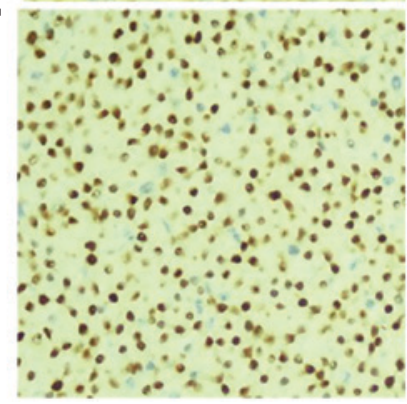

Figure 4. Histological examination of the neoplasm. (A) Histological section of the mass with hematoxylin and eosin staining. Immunohistochemical staining for (B) Ki-67, (C) CD38, (D) CD138, (E) light chain $\lambda$, and (F) multiple myeloma oncogene-1. The positive ratio of Ki-67 was $>30 \%$. Magnification, $\mathrm{x} 400$. CD, cluster of differentiation.
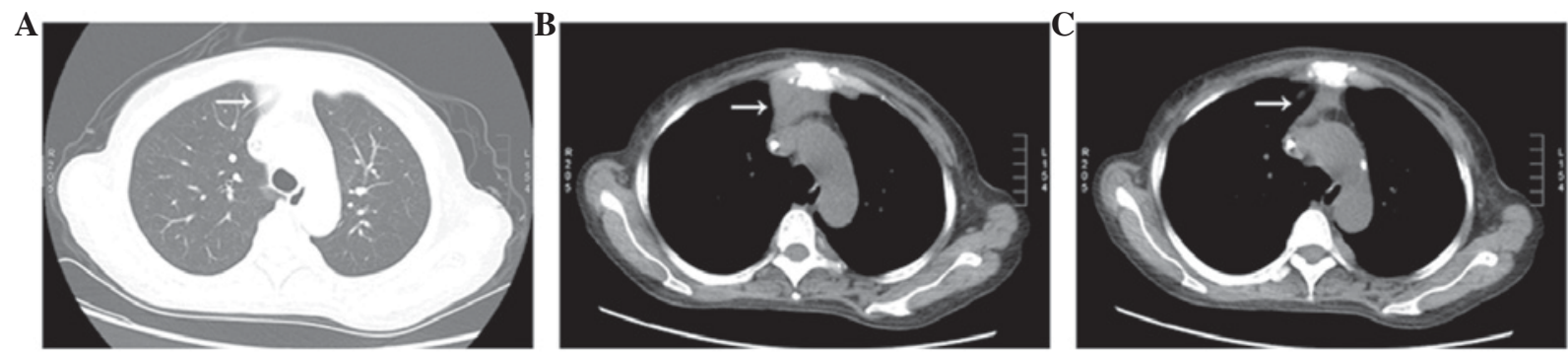

Figure 5. Chest computed tomography scan following the second cycle of chemotherapy. (A) Pulmonary and (B) mediastinal window prior to chemotherapy. (C) Mediastinal window following two cycles of chemotherapy.

115-150 g/l) level were decreased, whereas the other indices were normal. Serological tests showed that serum Ig and complement levels were normal ( $\mathrm{IgG}, 10.60 \mathrm{~g} / \mathrm{l}$; IgA, $0.74 \mathrm{~g} / \mathrm{l} ; \mathrm{IgM}, 0.23 \mathrm{~g} / \mathrm{l} ; \mathrm{C} 3,1.26 \mathrm{~g} / \mathrm{l}$; and $\mathrm{C} 4,0.35 \mathrm{~g} / \mathrm{l})$. The patient's serum $\beta 2$-microglobulin $(24.30 \mathrm{mg} / 1$; normal range, $0.7-1.8 \mathrm{mg} / \mathrm{l})$ and lactate dehydrogenase $(504 \mathrm{U} / 1$; normal range, normal range, 115-150 U/l) levels were significantly increased. Immunofixation electrophoresis revealed that monoclonal protein was negative in the serum, but LAM was positive in the urine. The level of KAP (1.60 g/l; normal range, 1.7-3.7 g/l) was slightly decreased, while the level of LAM (2.64 g/l; normal range, 0.9-2.1 g/l) was increased in the serum. In the 24-h urine analysis, the level of KAP was $0.60 \mathrm{mg}$ and the level of LAM was $2,360.00 \mathrm{mg}$. The patient's urine protein was $++(>1.0 \mathrm{~g} / \mathrm{l}$; normal range, $<0 \mathrm{~g} / \mathrm{l}$ ), according to the Durie-Salmon (DS) system and the International Staging System (ISS) (3).

Finally, after combining the results of the clinical manifestations, imaging diagnosis and laboratory examinations, including serum, urine, BM and biopsy pathology analysis, the tumor was diagnosed as a multiple myeloma of LAM type, DS stage IIA/ISS stage III.
Following the diagnosis, the patient was administered two courses of chemotherapy (10 mg epirubicin on days 1-4 intravenous drop infusion (ivd); $20 \mathrm{mg}$ dexamethasone on days 1-4 and 9-12 ivd; and $100 \mathrm{mg}$ oral thalidomide daily). The tumor on the right sternoclavicular junction reduced in size after chemotherapy (Fig. 5). However, the 24-h urine protein, KAP, LAM and $\beta 2$-microglobulin levels were $3.54 \mathrm{~g} / 24 \mathrm{~h}$, $27.94 \mathrm{mg} / \mathrm{h}, 2948 \mathrm{mg} / \mathrm{h}$ and $33.40 \mathrm{mg} / \mathrm{l}$, respectively, indicating no significant change compared with the levels prior to treatment. Meanwhile, the patient exhibited renal failure, most likely due to the kidney injury caused by the primary disease, but the condition improved after rehydration by diuretic therapy. Next, the patient was switched to one course of PAD chemotherapy [1.7 mg intravenous (iv) bortezomib on days $1,8,15$ and 22; $10 \mathrm{mg}$ epirubicin on days 1-4 ivd; and $20 \mathrm{mg}$ dexamethasone on days 1-2, 8-9, 15-16 and 22-23 ivd). The original tumor was significantly reduced in size after chemotherapy. In the 24-h urine analysis, the KAP and LAM levels were $40.30 \mathrm{mg} / \mathrm{h}$ and $748.80 \mathrm{mg} / \mathrm{h}$, respectively, indicating a $68 \%$ decrease in comparison to the values prior to treatment. The patient was administered one additional course of PAD chemotherapy. Currently, the patient has 
completed the second course of PAD chemotherapy with all indicators improved (Fig. 5).

\section{Discussion}

Clonal proliferation of malignant plasma cells in the BM is a hallmark of neoplastic plasma-cell disorder, which can be observed as either extraosseous extramedullary plasmacytoma (EMP) or MM. The current case is associated with MM. It has been reported that only 5\% of patients with EMP have coexistent MM (4). Extramedullary plasmacytoma is an uncommonly localized plasma cell malignancy representing $3 \%$ of plasma cell neoplasms. While $80-90 \%$ of EMP involves the submucosa of the upper aerodigestive, infiltration into the mediastinum, scrotum, dermis and retroperitoneum is extremely rare (5).

The anterior mediastinum is rarely involved in MM with extramedullary plasmacytomas, particularly in the thymus. The patient in the present case was hospitalized due to an egg-like mass protruding from the upper right chest wall, which had been misdiagnosed as a malignant thymoma. The mass was diagnosed by classical BM biopsy and IHC of this chest biopsy. The radiology and pathology results confirmed that the mass was filled with abnormal plasma cells and originated from the anterior mediastinum. This case was characterized by a clonal population of BM plasma cells that secrete a monoclonal paraprotein and an Ig free light chain (FLC). All these plasma cells were grouped in a clustered arrangement, and the ratio of primitive to naive plasma cells reached $57 \%$. The main diagnostic criteria of MM is a BM monoclonal plasma cell count of $\geq 10 \%$ is (6), and plasma cells are counted based on the expression of CD38 and CD138 (7). Neoplastic myeloma cells can be discriminated from reactive plasma cells by determining the expression of CD19 (8); reactive but not neoplastic plasma cells express CD19. In the present case, IHC demonstrated that CD38 and CD138 were highly expressed, and that CD19 was not expressed in the tumor. Meanwhile, LAM expression in the cytoplasm was also positive. Furthermore, there were other significant indicators, such as the degree of LAM in the urine and hemoglobin in the serum. These results supported the diagnosis of MM of LAM type.

Plasma cells arise from the B cells of the BM and produce Igs that form the normal humoral immune response systems of the body. The Ig molecule is composed of a heavy chain and a light chain. Plasma cells usually produce an excess of light chains that do not bind to heavy chains to form a complete Ig molecule, but instead enter the bloodstream as FLCs. Light chains can be deposited in any tissue of the body, including the skin, mucosa, fat tissues and other vital organs (9), which is termed amyloid light-chain amylosis (AL). AL can induce severe organ dysfunction, such as renal failure. It has been reported that up to $30 \%$ of MM patients may have subclinical amyloid deposits (6). However, amylosis was not observed in the present case. Additional biopsy results are required to confirm such an observation.

In the present case, the results from BM biopsy, laboratory examinations, local biopsy pathology and IHC confirmed the diagnosis of LAM-type MM with involvement of the thymus, which has rarely been reported. LAM-type MM plasma cells may invade the thymus through the bloodstream and become a large lesion, suggesting an effect of immune disturbance arising from an abnormal thymus gland.

Consequently, the present case could be distinguished from a case of thymoma. Thymomas and thymic carcinomas are epithelial tumors that arise from the thymus gland in the anterior mediastinum. Among the anterior mediastinal tumors, thymoma is the most frequently diagnosed, representing $20 \%$ of all mediastinal neoplasms in adults $(10,11)$. Although the precise incidence rate of thymomas is not well documented, it is estimated to be $0.15 / 100,000$ person-years (12). The rarity and morphological heterogeneity of thymomas have significantly contributed to the difficulty in behavioral prediction for these tumors. Thymomas are typically slow-growing tumors that spread by local extension. Moreover, thymoma metastases are typically confined to the pleura, pericardium or diaphragm, whereas extrathoracic metastases are uncommon (13).

While the present case was later confirmed to be MM with extramedullary plasmacytomas in the thymus, it was first diagnosed as basic MM. Plasmacytomas are formed from localized plasma cell accumulations that coalesce into distinct tumors. These tumors normally occur in the BM, but can occasionally be located at extramedullary sites, including the mediastinum. Extramedullary MM progression has consistently been associated with a poor disease prognosis. An increasing number of studies have shown that extramedullary progression is associated with secondary changes in the myeloma clone, poor prognostic histological and biological factors, aggressive disease progression and resistance to treatment (4). In the present case, following the first cycle of chemotherapy with epirubicin, dexamethasone and thalidomide, the patient showed signs of renal failure. Following the second cycle of chemotherapy, the patient recovered, with a reduction in the size of the tumor mass. It is believed that radiotherapy in combination with systemic chemotherapy is the best strategy for the treatment of plasmacytoma associated with MM (14). Therefore, the present patient will be administered radiotherapy to eliminate the tumor mass.

The present study reports a rare case of MM with thymus involvement. The diagnosis was based on the results of BM analysis, laboratory examinations, local biopsy pathology and IHC. The study suggests that thymus involvement in MM may be consistent with an effect of immune disturbance arising from an abnormal thymus gland. Based on this case, we propose that, in the clinic, extramedullary plasmacytoma in the thymus should be carefully distinguished from thymoma.

\section{Acknowledgements}

The present study was supported by the National Natural Science Foundation of China (grant no. 81372870 awarded to Ke-Wei Ma).

\section{References}

1. Palumbo A and Anderson K: Multiple myeloma. N Engl J Med 364: 1046-1060, 2011.

2. Raab MS, Podar K, Breitkreutz I, Richardson PG and Anderson KC: Multiple myeloma. Lancet 374: 324-339, 2009.

3. Stewart AK and Fonseca R: Review of molecular diagnostics in multiple myeloma. Expert Rev Mol Diagn 7: 453-459, 2007. 
4. Masood A, Hudhud KH, Hegazi A and Syed G: Mediastinal plasmacytoma with multiple myeloma presenting as a diagnostic dilemma. Cases J 1: 116, 2008

5. Ahnach M, Marouan S, Rachid M, et al: Extramedullary plasmocytoma relapsing at different sites: An unusual presentation. Pan Afr Med J 14: 34, 2013.

6. Eslick R and Talaulikar D: Multiple myeloma: From diagnosis to treatment. Aust Fam Physician 42: 684-688, 2013.

7. Cho YU, Park CJ, Park SJ, et al: Immunophenotypic characterization and quantification of neoplastic bone marrow plasma cells by multiparametric flow cytometry and its clinical significance in Korean myeloma patients. J Korean Med Sci 28: 542-549, 2013.

8. Hosen N: Multiple myeloma-initiating cells. Int J Hematol 97: 306-312, 2013.

9. Siragusa S, Morice W, Gertz MA, et al: Asymptomatic immunoglobulin light chain amyloidosis (AL) at the time of diagnostic bone marrow biopsy in newly diagnosed patients with multiple myeloma and smoldering myeloma. A series of 144 cases and a review of the literature. Ann Hematol 90: 101-106, 2011.
10. Mullen B and Richardson JD: Primary anterior mediastinal tumors in children and adults. Ann Thorac Surg 42: 338-345, 1986.

11. Strollo DC, Rosado de Christenson ML and Jett JR: Primary mediastinal tumors. Part 1: Tumors of the anterior mediastinum. Chest 112: 511-522, 1997.

12. Gokmen-Polar Y, Cook RW, Goswami CP, et al: A gene signature to determine metastatic behavior in thymomas. PLoS One 8: e66047, 2013.

13. Regnard JF, Magdeleinat P, Dromer C, et al: Prognostic factors and long-term results after thymoma resection: A series of 307 patients. J Thorac Cardiovasc Surg 112: 376-384, 1996.

14. Holstein SA, Liu H and McCarthy PL: Multiple myeloma. Hematol Oncol Clin North Am 28: 1113-1129, 2014. 